\title{
Fertilité après radiothérapie
}

\author{
A. Fignon*, S. HamamaH*, G. Calais** \\ *Département de Gynécologie-Obstétrique, Reproduction Humaine et Médecine Fotale; \\ **Service de Radiothérapie - CHRU Bretonneau, 2 Boulevard Tonnellé 37044 Tours Cedex
}

\section{RESUME}

La radiothérapie fait partie de l'arsenal thérapeutique de la cancérologie. De jeunes patients sont traités pour maladie de Hodgkin ou pour tumeurs testiculaires entraînant des troubles de la fertilité.Notre article se propose de faire la revue de la littérature concernant la fertilité après radiothérapie et d'insister sur l'information des patients ainsi que la prévention.

Mots clés : fertilité, radiothérapie, tumeurs testiculaires, maladie de Hodgkin.

\section{INTRODUCTION}

Les avancées thérapeutiques en oncologie permettent d'espérer des survies prolongées et des guérisons chez un nombre important de patients traités par radiothérapie. Les progrès sont d'autant plus sensibles que les sujets sont jeunes. Nous envisagerons donc les paramètres influençant la fertilité d'un couple dont l'un des membre a été traité par radiothérapie.

Les données du National Cancer Institute (NCI) montrent que $65 \%$ des enfants américains, qui ont été traités pour cancer entre 1983 et 1989 , sont vivants plus de 5 ans après le traitement, contre seulement $28 \%$ il y a 20 ans [1]. En 1984, 45000 adultes américains avaient été traités pour cancer pendant leur enfance [2]. Les améliorations pronostiques concernent également l'adulte jeune, notamment en matière de maladie de Hodgkin et carcinome testiculaire. Avec l'augmentation des survivants à long terme, les études portant sur les complications tardives deviennent possibles. L'analyse des conséquences de la radiothérapie sur la fertilité s'avère néanmoins d'approche difficile [3], si la fertilité est estimée en terme de grossesse menée à terme. En effet, même en cas de fertilité conservée, une conception pourra être différée ou évitée par crainte d'une rechute, par inquiétude quant à la santé de l'enfant. La nécessité d'un recul important sur grandes cohortes de patients complique la méthodologie, si bien qu'aucune étude prospective n'a été publiée à ce jour.

Toute radiothérapie ne s'accompagnant pas de stérilité, le pronostic gonadique ne peut être toutefois établi qu'en fonction du sexe des patients, de la pathologie traitée (baisse de fertilité au cours de la maladie de Hodgkin ou du cancer du testicule), des drogues associées employées, de leur doses, et à une moindre degré de l'âge des patient(e)s.

Chez la femme, même si la procréation reste possible, la fenêtre de fertilité (période séparant la puberté de la ménopause) peut être réduite par la diminution du stock folliculaire [4]. Dans une série portant sur le pronostic gynécologique de patientes traitées par alkylants et radiothérapie entre 13 et 19 ans, le risque de ménopause précoce était 27,4 fois plus élevé lorsque ces patientes atteignaient la tranche d'âge 21- 
25 ans par rapport à la population normale. Ce risque diminuait à 4,6 fois pour la tranche d'âge 26-30 ans. Par ailleurs, l'exposition aux alkylants seuls conférait un risque relatif de ménopause précoce de 9,1 aux patientes de 21-25 ans, contre 3,7 en cas de radiothérapie exclusive et de 1,1 après chirurgie seule. Ce risque augmentait avec les doses administrées et avec l'âge des patientes $[4,5]$.

Chez l'homme, plus que le type de cancer, ce sont les médicaments utilisés et leurs dosages qui génèrent le risque de stérilité. L'association d'une irradiation sous-diaphragmatique et d'une chimiothérapie est fortement synergique [3].

Même lorsqu'un des membres du couple soumis à une thérapeutique anticancéreuse garde soit des perturbations du cycle menstruel soit une oligozoospermie, tout risque de grossesse ne peut être écarté en raison du risque d'ovulation inopinée lors d'une aménorrhée apparemment installée [6], de récupération tardive de la fonction spermatocytaire, ou de la possibilité de procréation même en cas d'oligospermie sévère [5]. Les couples ne souhaitant pas avoir d'enfant doivent donc être avertis de la nécessité d'une contraception.

Les couples désirant une grossesse doivent être rassurés quant au risque de tératogénicité et de cancer induit dans la descendance, dans la limite des connaissances actuelles. Cette opinion, doit être néanmoins nuancée lorsque la tumeur traitée est à composante familiale possible (tumeur de Wilms, rétinoblastome). Un caryotype à la recherche d'une monosomie 11p-ou 13q-doit être alors effectuée [5]. Dans les autres situations, le caryotype n'est d'aucune utilité.

\section{TOXICITE DE LA RADIOTHERAPIE}

La radiothérapie joue un rôle important dans le traitement de nombreux cancers, en particulier les lymphomes et les sarcomes.
Elle est le plus souvent associée aux autres thérapeutiques comme la chimiothérapie, mais elle peut être parfois utilisée comme thérapeutique exclusive. Il est souvent très difficile de faire la différence entre la toxicité sur les gonades directement liée à la radiothérapie et celle provoquée par l'association radiothérapie et chimiothérapie. Par ailleurs les données concernant les effets de la radiothérapie sur la fonction gonadique sont peu abondantes en comparaison avec l'abondance de la littérature sur la toxicité de la chimiothérapie.

Le testicule est hautement radiosensible, très vraisemblablement à cause de la rapidité de division cellulaire de l'épithélium germinal. Des données anciennes ont permis d'établir des courbes de radiosensibilité du testicule humain. Une diminution notable mais transitoire de la production de sperme est décelable pour des très faibles doses de l'ordre de 15 cGy. Des aspermies transitoires ont été rapportées pour des doses de 50 cGy. Pour des doses de 200 à 300 cGy il faut 3 ans pour recouvrer une production de sperme normale. Entre 400 et $600 \mathrm{cGy}$ l'intervalle peut être de 5 ans. La stérilité est pratiquement constante au delà de 600 cGy [1-3]. Une évaluation récente de la fonction testiculaire après traitement pour maladie de Hodgkin montre que la fonction gonadique est compromise pour des faibles doses de l'ordre de 50 cGy et que pour des doses cumulées de l'ordre de 200 cGy un dysfonctionnement testiculaire persiste plus de 3 ans [4]. Peu de données sont disponibles sur la toxicité directe de la radiothérapie sur le testicule. Cependant des doses élevées de l'ordre de 2400 cGy délivrées directement sur le testicule dans le cadre du traitement de rechute de leucémie aiguë lymphoblastique provoquent une atrophie testiculaire avec un hypogonadisme clinique.

L'ovaire est aussi un organe très radiosensible. Le problème est d'autant plus complexe chez la femme car l'ovaire est situé à proximité de chaînes ganglionnaires lym- 
phatiques importantes et il est donc souvent situé directement dans le faisceau d'irradiation ou à proximité immédiate. $\mathrm{Au}$ delà de 150 cGy les aménorrhées sont fréquentes et quasi constantes à partir de 600 à $700 \mathrm{cGy}$ [6]. L'âge est un facteur important puisque dans la maladie de Hodgkin, après irradiation lymphoïde totale, $70 \%$ des femmes de moins de 20 ans gardent des cycles réguliers alors que seulement $20 \%$ conservent une fonction ovarienne normale après 30 ans [7].

La toxicité de la radiothérapie sur le testicule ou l'ovaire peut être diminuée en utilisant :

- Des protections testiculaires lorsque le faisceau est dirigé sur la région testiculaire mais aucune étude n'a évalué avec précision l'intérêt de cette méthode. De plus la toxicité de la radiothérapie sur le testicule est surtout due au rayonnement diffusé qui échappe à toute tentative de protection.

- L'utilisation de caches testiculaires dans le traitement des séminomes ou des lymphomes permet d'abaisser à moins de $10 \%$ de la dose prescrite la dose délivrée au testicule $[8,9]$.

- La transposition ovarienne qui consiste à déplacer chirurgicalement les ovaires avant une irradiation pelvienne permet de réduire considérablement la dose ovarienne. Avant traitement pour maladie de Hodgkin, il est souhaitable de les positionner sur la ligne médiane, derrière l'utérus. Cette intervention peut se faire soit par cœlioscopie soit par laparotomie. Elle est proposé aux patientes de moins de 40 ans. Une protection médiane de double épaisseur sera mise en place pour atténuer le plus possible le rayonnement transmis [10].

\section{PRONOSTIC DE LA GROSSESSE}

Le risque doit être envisagé sous deux aspects différents : le risque génétique à long terme et le risque tératogène immédiat.

\section{Effets Génétiques}

Les agents utilisés pour traiter les cancers sont conçus pour interférer avec l'ADN, le métabolisme et la division cellulaire et il y a donc de bonnes raisons de penser qu'ils sont capables d'induire des mutations et des maladies génétiques.

Sur des modèles animaux ont été établies des courbes dose-réponse entre irradiation et risque génétique sur les cellules germinales. Elles servent de référence pour les normes de radioprotection mais elles ne reflètent pas la sensibilité aux radiations ionisantes des cellules germinales humaines [11]. Les données proviennent des observations humaines et des expérimentations animales. Ainsi les survivants des explosions atomiques au Japon ont été particulièrement étudiés en ce qui concerne le risque génétique dans leur descendance et les données tendent à prouver que les cellules germinales humaines sont beaucoup plus tolérantes aux radiations ionisantes que celles des animaux de laboratoires. Dans ces populations les effets cancérigènes ont été prouvés de façon indiscutable, par contre aucune différence statistiquement significative n'a été observée pour les effets génétiques, même pour des doses élevées [12]. Des données récentes suggèrent que la dose nécessaire pour doubler le taux de mutations spontanées est 5 fois plus élevée chez l'homme que chez la souris $[13,14]$.

Les effets génétiques ne sont pas spécifiques : les mutations liées aux radiations ionisantes ne sont pas différentes de celles provoquées par d'autres agents. Ils apparaissent souvent tardivement ; les mutations dominantes se manifestent à la première génération, mais les mutations recessives ne se manifestent, si elles le font, qu'après plusieurs générations. Ils surviennent de façon aléatoire. Chez l'animal plus le débit de dose est élevé et plus le fractionnement est faible plus le risque de mutation est élevé [15]. 


\section{Effets Tératogènes}

Ce sont les anomalies ou malformations provoquées par l'action des radiations ionisantes sur l'embryon ou le fotus au cours de la grossesse.

Aucune lésion n'a été constatée pour des doses inférieures à $0.2 \mathrm{~Gy}$, mais on ne peut affirmer qu'il y ait un seuil. La radiosensibilité varie suivant le stade du développement de l'embryon : elle est maximale au stade de l'organogénèse. Les effets tératogènes sont indépendants de l'agent s'ils agissent au même stade de la période d'organogénèse. Ils sont irréversibles.

L'utilisation de la radiothérapie pendant la grossesse n'est pas formellement contreindiquée notamment après la fin du premier trimestre. La décision doit être bien entendu prise en fonction du stade de la grossesse et du risque carcinologique. Des protections plombées efficaces peuvent être mises en place et un monitorage exact de la dose délivrée au foetus doit être fait. Il est habituel de considérer que, lorsque la dose délivrée entre la 8ème et la 15ème semaine de gestation dépasse 100 rads, le risque de malformation du système nerveux central est important [16]. Dans le cadre du traitement de la maladie de Hodgkin une radiothérapie sus diaphragmatique peut être effectuée à la dose de 30 à $35 \mathrm{~Gy}$ avec une protection fotale efficace. Par contre traiter par curiethérapie utéro vaginale à "faible dose" un cancer du col utérin limité pendant la grossesse en différant la radiothérapie pelvienne après le terme de la grossesse doit être proscrit car inefficace sur le plan carcinologique et à très haut risque de complication fotale [17].

\section{Fertilité, radiothérapie et greffe de moelle osseuse}

Trente sept grossesses ont été rapportées chez 27 femmes âgées de 17 à 36 ans, traitées par allogreffe de moelle osseuse pour aplasie médullaire (15 femmes) ou pour leu- cémie aiguë ( 12 femmes). Toutes avaient reçu une conditionnement par cyclophosphamide seule à forte dose $(120 \mathrm{mg} / \mathrm{kg})(15$ femmes), ou combinée à une irradiation corporelle totale ( 10 femmes) ou à du melphalan (2 femmes). Les grossesses sont survenues dans un délai de 8 à 96 mois après la greffe. Après cyclophosphamide seule, 18 grossesses chez 15 femmes ont permis la naissance de 13 enfants vivants contre 5 avortements (3 spontanés et 2 thérapeutiques). Trois grossesses ont été menées à terme près cyclophosphamide-melphalan. Après irradiation corporelle totale, 12 grossesses dont 1 en cours, 6 enfants vivants et 5 avortements (4 spontanés et 1 thérapeutique) sont rapportées. Deux grossesses sont survenues chez des femmes aménorrhéiques, sans passer par la fécondation in vitro.

\section{PROTECTION DE LA FERTILITE}

Il est nécessaire avant toute radiothérapie d'évaluer le risque d'irradiation des gonades.Lorsque cela est possible, il est nécessaire d'utiliser les drogues les moins nocives pour les gonades et de mettre en place un système de protection des gonades si le traitement entraîne une gonadotoxicité (Exemple : le MOPP est responsable de stérilité irréversible). Des prélèvements de sperme seront effectués et congelés. Toutefois la congélation entraîne elle même une altération du sperme. Bientôt ce problème sera minimisé par les progrès de la procréation médicalement assistée notamment l'ICSI [18].

\section{PROGRAMMATION DE LA GROSSESSE}

La date de la grossesse chez un couple antérieurement traité par radiothérapie doit être discutée en fonction du pronostic de la tumeur, de sa compatibilité avec une grossesse (sein). En général un délai de trois ans est requis entre la fin du traite- 
ment et le début d'une grossesse. Ce délai ne doit pas être exagérément augmenté, à cause du risque de ménopause précoce induit par le traitement.

Lorsqu'une grossesse désirée ne peut être obtenue après les délais habituels et lorsqu'un cycle menstruel persiste chez la femme, une prise en charge spécialisée en vue d'une éventuelle fécondation in vitro doit être envisagée [19].

\section{LA SURVEILLANCE DE LA GROSSES- SE APRES RADIOTHERAPIE}

La prise en charge est tout à fait différente suivant le type de tumeur traitée au préalable, l'angoisse du couple ou au contraire la joie du début de grossesse ressentie comme une revanche sur le sort, la proximité ou non de la thérapeutique, le partenaire (l'homme ou le femme ) ayant reçu le traitement. La prise en charge d'une grossesse après radiothérapie doit débuter avant une conception pour faire la part des possibilités de grossesse et mettre en place la surveillance.

Il est licite à notre avis que ce type de grossesse soit pris d'emblée en charge par le spécialiste responsable de l'accouchement en relation étroite avec le médecin généraliste qui s'occupe habituellement du couple. Des contacts étroits seront noués avec les responsables du traitement initial afin d'avoir un suivi pluridisciplinaire. La consultation doit être précédée si possible du courrier du spécialiste oncologique et radiothérapeute ayant réalisé la thérapeutique initiale. A défaut de document l'interrogatoire doit débuter comme pour une grossesse normale et attendre les questions qui angoissent le couple. En général, elles surviennent rapidement: Le couple est inquiet du risque tératogène car on lui a expliqué que la radiothérapie ou la chimiothérapie pouvait abîmer les chromosomes des cellules gonadiques avec un risque de transmission. Il s'inquiète du risque poten- tiel de récidive et des possibilités de complications de la grossesse liées au traitement antérieur. Il faut savoir dédramatiser ce début de grossesse. Souvent elle a été acceptée après information des risques encourus voir après un conseil génétique ou une étude de la pharmacovigilance.

En effet le potentiel mutagène de la radiothérapie et de la chimiothérapie est rendu responsable de malformations fœtales, de risque de développement d'un cancer, ou de risque d'avortement. Un certain nombre d'articles dans la littérature sont encourageants quand à la prise en charge de ces grossesses.

Ainsi, il ne semble pas exister d'augmentation des malformations dans la descendance des patients survivants après une thérapeutique mutagène comme la chimiothérapie comprenant des alkylants ou une irradiation gonadique. Il n'y a pas non plus de modifications du sex-ratio.

\section{CONCLUSION}

La protection de la fertilité doit être un souci permanent du radiothérapeute et sera basé sur la mise en place de techniques de radioprotection et sur l'utilisation des drogues les moins toxiques possibles sans altérer les chances de survie.

Le pronostic gonadique devrait, dans la mesure du possible, être expliqué aux patient(e)s, ou aux parents des enfants atteints de tumeur maligne, dès le début de la maladie. En fait bien souvent, ce problème est relégué au second plan, soit par oubli de formulation de la part du médecin, soit du fait du choc émotionnel lié à l'annonce du diagnostic. La fertilité future est une question d'autant plus importante à aborder que la discussion devra obligatoirement envisager l'opportunité d'une cryoconservation de sperme avant toute chimiothérapie, chez les hommes en âge et désireux de procréer, lorsque le traitement risque d'avoir des effets délétères sur la fonction 
testiculaire.La cryoconservation était souvent limitée avec une mauvaise qualité en décongélation (50\% dans les cancers testiculaires et $23 \%$ dans las maladies de Hodgkin).Une amélioration des performances est à envisager dans l'avenir grâce aux progrès de l'assistance médicale à la procréation (ICSI).

\section{REFERENCES}

1. Ash P. : The influence of radiation on fertility in man. Br J Radiol, 1980 ; 53 : 271-278.

2. Hahn EW, Feingold SM, Simpson L et Al. : Recovery of aspermia induced by low dose radiation in seminoma patients. Cancer, $1982 ; 50: 337-340$.

3. Shapiro E, Kinsella TJ, Makoch RW e't al. : Effects of fractinated irradiation on endocrine aspects of testicular function. $\mathrm{J}$ Clin Oncol, 1985 ; $3: 1232-1239$.

4. Meistrich ML, VON BEeK ME. : Radiation sensitivity of the human testis. Adv Radiat Biol, $1990 ; 14$ : 227-268.

5. Bralner R, Czernichow P, Cramer P et al. Leydig cell function in children after direct testicular irradiation for acute lymphoblastic leukemia. N Engl J Med, 1983 ; 309 : 25-28.

6. Horning SJ, Hoppe RT, Kaplan HS ET AL. : female reproductive potential after treatment for hodgkin disease. N Engl J Med, 1982 ; 304 : 1377-1382.

7. Thomas PR, Winstantly D, Pecham MJ et al. : reproductive and endocrine function in patients with hodgkin disease. Effects of oophoropexy and irradiation. Br J Cancer, $1976 ; 33: 226$ 231.

8. FraAs BA, Kinsella TJ, Glatstein EJ. : Peripheral dose to the testis. The design and use of a practical and effective gonadal shield. Int $J$ Radiat Oncol Biol Phys, 1985 ; 11 : 609-615.

9. PfnRick TJ, Hoppe RT. : Recovery of spermatogenesis following pelvic radiotherapy for hodgkin's disease. Int J Radiat Oncol Biol Phys, 1986; 12 : 117-121.
10. Ray GR, TRLebloon HW, EnRight LP et al : Oophoropexy : A means of preserving ovarian function following pelvic meagavoltage radiotherapy for hodgkin's disease. Radiology, $1970 ; 96: 175-180$.

11. SHULı, WJ. : Reproductive problems : Fertility teratogenesis and mutagenesis. Arch Envir Health, $1984 ; 39: 207-212$

12. MARX JL. : Lower radiation effect found. Science, $1988 ; 241: 1286$.

13. NEEL JV. Genetic effects of atomic bombs. Science, $1981 ; 213: 1205$.

14. Nril $\mathrm{JV}$, Lrwis SE. : The comparative radiation genetics of humans and mice. Ann Rev Genet, $1990 ; 24: 327-362$.

15. RLSSEI, WL, Russel LB. : Radiation induced genetic damage in mice. In Progress in nuclear energy, Bugher JC (eds), London, Pergamon Press, 1979; pp.179-188.

16. Ronderos A. : Fetal tolerance to radiation. Radiology, $1961 ; 76: 454-456$.

17. Stralss A. : Irradiation of carcinoma of the cervix uteri during pregnancy. AJR, $1940 ; 43: 552$.

18. Mriron D, Schenker J.G. : Cancer and male fertility. Human Reprod., 1995, $10: 2017-2022$.

\section{ABSTRACT}

Fertility after radiotherapy
A. Fignon, S. Hamamah, G. Callais

It is now well established that fertility of of young patients is compromised after the radiotherapy treatment for malignant diseases. This paper summarizes the main data concerning the fertility of young patients after radiotherapy treatment. The information of the patients as well as the prevention are the subject of this review.

Key Words : Testes, fertility, radiotherapy, cancer. 